\title{
Improved Superorthogonal Codes Through Generalized Rotations
}

\author{
Mohammad Janani, Ahmadreza Hedayat, Member, IEEE, and Aria Nosratinia, Senior Member, IEEE
}

\begin{abstract}
Concatenation of orthogonal space-time block codes (OSTBC) with an outer trellis has led to simple and powerful codes, known as superorthogonal codes or space-time block trellis-coded modulation. In this letter, we generalize these codes by finding new code supersets and corresponding set partitioning, resulting in improved coding gain. We provide design guidelines for the labeling of the generalized code trellises and demonstrate the gains by several example designs for two and four transmit antennas.
\end{abstract}

Index Terms-Multiple-input multiple-output (MIMO), spacetime coding, superorthogonal codes.

\section{INTRODUCTION}

$\mathbf{E}$ VER SINCE the first works on space-time coding appeared, the research community has been seeking space-time codes (STCs) with good complexity/performance tradeoff. Thus, as in other branches of coding, a continual effort has been made to find codes with a structure that allows simple decoding, while maintaining good performance. An attractive tradeoff between structure and performance is made possible by a concatenation of orthogonal space-time block codes (OSTBCs) with a trellis, which provides high performance at a relatively small computational cost. The contribution of this letter consists of generalizations, improvements, and systematic code design for this new class of codes.

A brief background of work in this area follows. Jafarkhani and Seshadri proposed codes consisting of an OSTBC concatenated with a blockwise trellis, known as superorthogonal STCs [1] (SOSTCs). The design process is similar to the trellis-coded modulation (TCM) of Ungerböck: the codebook of the orthogonal block codes is expanded and then partitioned into sets with suitable distance properties. Then the trellis is labeled appropriately with the set partitions. At the same time, Siwamogsathan and Fitz [2] independently proposed similar trellis-block codes, with an approach that is somewhat more general. Both of these codes must be handcrafted.

In this letter, we revisit the problem of building trellises over STBCs and propose a generalized mapping of modulations to the antenna signals that leads to better codes. We provide design criteria for the generalized block-trellis codes. The proposed systematic method for the design of OSTBCs ensures that good codes are not overlooked. The search complexity is reduced by observing certain properties of trellises over OSTBCs.

Paper approved by H. El Gamal, the Editor for Space-Time Coding and Spread Spectrum of the IEEE Communications Society. Manuscript received November 15, 2004; revised February 15, 2006. This work was supported in part by the National Science Foundation under Grant CNS-0435429. This paper was presented in part at the Globecom Conference, 2004.

M. Janani and A. Hedayat are with Navini Networks, Richardson, TX 75082 USA (e-mail: janani@utdallas.edu; hedayat@ieee.org).

A. Nosratinia is with the Department of Electrical Engineering, University of Texas at Dallas, Richardson, TX 75083 USA (e-mail: aria@utdallas.edu).

Digital Object Identifier 10.1109/TCOMM.2007.894107
We use the following notation. Uppercase bold letters denote matrices, for example, codewords are denoted with $\mathbf{X}, \mathbf{Y}, \mathbf{Z}$, and unitary transforms with $\mathbf{U}, \mathbf{V}, \mathbf{W}$, which we concisely (but not entirely accurately) refer to as "rotations" in the following. Script letters denote sets of codewords, e.g., $\mathcal{T}, \mathcal{S}$. Subscripts are used to denote set partitioning and assignment of codeword sets to trellis states and trellis branches. In particular, $\mathcal{T}=\bigcup_{i} \mathcal{T}_{i}$, where $\mathcal{T}_{i}$ is a set partition for trellis state $i$, and $\mathcal{T}_{i}=\bigcup_{j} \mathcal{T}_{i, j}$, where $\mathcal{T}_{i, j}$ denotes the set of codewords assigned to the trellis branch going from state $i$ to state $j$. For convenience, we define the multiplication of a set and a matrix, for example, $\mathcal{T}_{0} \mathbf{U}$ as a new set whose members are the members of $\mathcal{T}_{0}$, each multiplied by $\mathbf{U}$. The function $\mathrm{D}(\cdot, \cdot)$ computes the minimum distance between two sets of codewords. With an abuse of notation, we may see a codeword as one of the arguments of this function, which should be interpreted as the set consisting of that single codeword.

The system model consists of a multiple-input multiple-output (MIMO) system with $L_{t}$ transmit and $L_{r}$ receive antennas. The overall code is a concatenation of a multiple trellis-coded modulation (MTCM) outer code and an orthogonal space-time block (OSTB) inner code. To each state of the trellis code $N_{B}$, OSTB codewords of size $T \times L_{t}$ are assigned. Therefore, the overall rate of the code is $\log _{2}\left(N_{B}\right) / T$.

A flat-fading channel is assumed, where the channel gains are constant during each fade interval and independent in successive intervals. The received signal, denoted by a $T \times L_{r}$ matrix $\mathbf{R}$, after matched filtering has the following form: $\mathbf{R}=$ $\sqrt{\rho} \mathbf{X H}+\mathbf{N}$. The average received signal-to-noise ratio (SNR) per antenna shown by $\rho$. The matrix $\mathbf{X}$ is an OSTB codeword of size $T \times L_{t}$. The channel matrix $\mathbf{H}=\left\{h_{i j}\right\}$ has the size of $L_{t} \times L_{r}$, where $h_{i j}$ is the fading channel coefficient between the $j$ th received antenna and the $i$ th transmit antenna. The additive white Gaussian noise (AWGN) is shown by the matrix $\mathrm{N}$. The receiver employs a maximum-likelihood (ML) decoder with perfect knowledge of channel state information (CSI).

\section{TRellis Design fOR BlOck STCS}

We follow the well-known trellis design principles developed by Ungerböck and applied to OSTBCs in [1], [2]. Ungerböck extended the original constellation set into a larger codebook (a superset), and each subset of the expanded codebook is called a subcode. Subcodes are designed and allocated to trellis branches in a manner that maximizes the performance of the code.

In the context of OSTBCs, the extension of the original codebook is accomplished via transformations $\mathbf{U}_{i}$. Each trellis state is allocated one rotation of the codebook $\mathcal{T}_{i}=\mathcal{T}_{0} \mathbf{U}_{i}$. Then, within each trellis state, we partition the codebook $\mathcal{T}_{i}=\mathcal{T}_{i, 0} \cup$ $\cdots \cup \mathcal{T}_{i, M-1}$ into subsets each assigned to a trellis transition, where $M$ is the number of connected states. Thus, $\mathcal{T}_{i, j}$ is the set of codewords assigned to the trellis branch that connects state $i$ to state $j$. If a transition does not have parallel branches, 


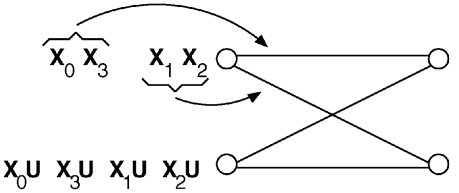

(a)

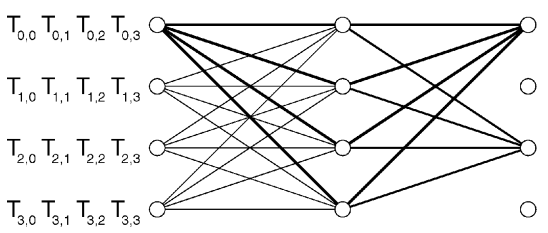

(b)

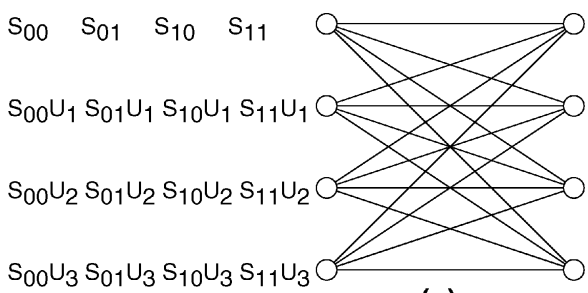

(c)

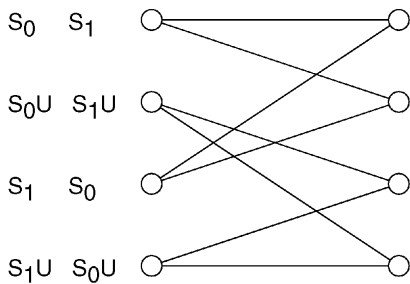

(d)

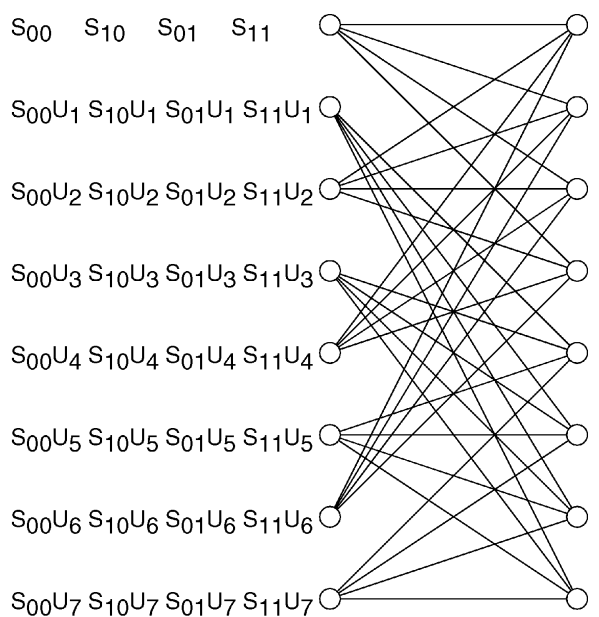

(e)

Fig. 1. (a) Two-state trellis code for $L_{t}=2 . \mathbf{X}_{i}, i=0,1,2,3$, are defined in (2), and $\mathbf{U}=\operatorname{diag}\left(e^{j \pi / 2}, e^{j 3 \pi / 2}\right)$. (b) Fully connected trellis. The trellises of (c) 4-state BPSK, (d) 4-state QPSK, and (e) 8-state QPSK codes. In parts (c)-(e), we follow the signaling notation of [1] for set partitions.

$\mathcal{T}_{i, j}$ will consist of one codeword; otherwise, it will have more than one codeword. The design question boils down to finding good transformations $\mathbf{U}_{i}$. Our contribution consists of generalizations, as well as providing design criteria that systematize code design, thus leading to improvements over existing codes.

The process can be made more clear by an example. Consider a system with two transmit antennas, with the following orthogonal block code due to Alamouti:

$$
\mathbf{X}\left(s_{0}, s_{1}\right)=\left(\begin{array}{cc}
s_{0} & s_{1} \\
-s_{1}^{*} & s_{0}^{*}
\end{array}\right)
$$

which, with binary phase-shift keying (BPSK) modulation, has the four codewords

$$
\begin{array}{ll}
\mathbf{X}_{0}=\left(\begin{array}{cc}
1 & 1 \\
-1 & 1
\end{array}\right) & \mathbf{X}_{1}=\left(\begin{array}{cc}
-1 & 1 \\
-1 & -1
\end{array}\right) \\
\mathbf{X}_{2}=\left(\begin{array}{cc}
1 & -1 \\
1 & 1
\end{array}\right) & \mathbf{X}_{3}=\left(\begin{array}{cc}
-1 & -1 \\
1 & -1
\end{array}\right) .
\end{array}
$$

The four codewords of the above block code form the subcode $\mathcal{T}_{0}$, which we assign to state 0 of the trellis [see Fig. 1(a)]. For the other state of the trellis, we use a different set of codewords obtained using a transformation $\mathbf{U}=\operatorname{diag}\left(e^{j \pi / 2}, e^{j 3 \pi / 2}\right)$, i.e., the four codewords used in state 1 are $\mathcal{T}_{1}=\left\{\mathbf{X}_{i} \mathbf{U}, i=0, \ldots, 3\right\}$. We denote $\mathcal{T}_{1}=\mathcal{T}_{0} \mathbf{U}$. For the example above, the rotation $\mathbf{U}$ suggested by our design procedure results in $1 \mathrm{~dB}$ gain compared with similar codes from [1] (see Fig. 2).

From this example, it is seen that our unitary transforms, unlike [1], generate modulation symbols that may not be in the original constellation. This is similar to the constellation expansion of Ungerböck [3], and much like that case, the peak-to-average power ratio remains the same and detector complexity is not much affected, because for each trellis state, only a smaller (original) constellation is transmitted.

We now proceed to analyze the structure of the rotation matrices $\mathbf{U}$. The per-antenna power constraint implies that the matrices $\mathbf{U}$ must be not only unitary, but also either diagonal or antidiagonal, as shown below. Because either will serve our purposes, we choose diagonal matrices in the following.

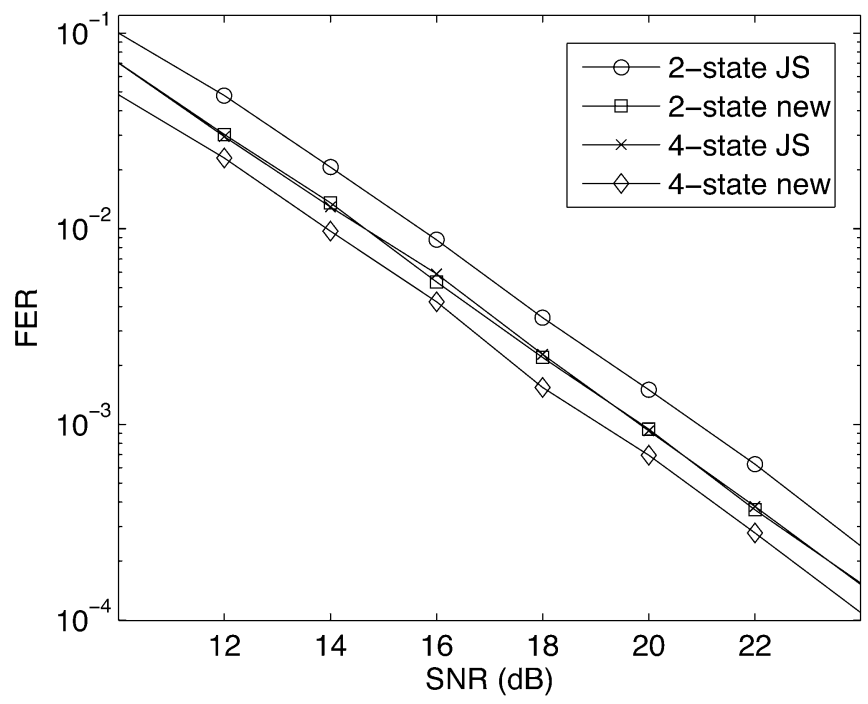

Fig. 2. $L_{t}=2$, two- and four-state BPSK codes in slow fading.

Lemma 1: Assuming equal transmit power from all antennas, the transformation matrices $\mathbf{U}$ used for expanding codeword sets must be either diagonal or antidiagonal.

Proof: Transform one codeword to anther via $\mathbf{Y}=\mathbf{X U}$, i.e.,

$$
\mathbf{Y}=\mathbf{X}\left(\begin{array}{ll}
a & b \\
c & d
\end{array}\right)=\left(\begin{array}{cc}
a s_{0}+c s_{1} & b s_{0}+d s_{1} \\
-c s_{1}^{*}+d s_{0}^{*} & -b s_{1}^{*}+d s_{0}^{*}
\end{array}\right)
$$

Because this must be true for any two modulation symbols $s_{0}$ and $s_{1}$, the per-antenna power constraint yields that either $c=$ $b=0$ and $|a|=|d|=1$, or $a=d=0$ and $|c|=|b|=1$. We have two acceptable representations, thus without loss of generality, we can choose the diagonal transform between two codewords, namely, $\mathbf{U}=\operatorname{diag}\left(e^{j \theta_{1}}, e^{j \theta_{2}}\right)$.

The next step is set partitioning and trellis labeling. Set partitioning requires a distance measure. Following [1], we introduce the coding gain distance (CGD). For two codewords $\mathbf{X}$ and $\mathbf{Y}$, construct $\mathbf{A} \triangleq(\mathbf{X}-\mathbf{Y})(\mathbf{X}-\mathbf{Y})^{H}$, and then define CGD 
$=\operatorname{det}(\mathbf{A})$. By extension, the minimum CGD of a codebook $\mathcal{T}$ is defined as the minimum of CGD of all nonidentical codeword pairs in $\mathcal{T} \times \mathcal{T}$. Similarly, the distance between two codebooks $\mathcal{T}, \mathcal{S}$ is $\mathrm{D}(\mathcal{T}, \mathcal{S})=\min \operatorname{det}(\mathbf{A}(\mathbf{X}, \mathbf{Y}))$, where the minimization is over all pairs $(\mathbf{X}, \mathbf{Y}) \in \mathcal{T} \times \mathcal{S}$.

\section{A. Reduced-Complexity Code Design}

Both the set partitioning and index assignment require CGD calculations. A complexity problem arises partially from the fact that our overall codes are not only nonlinear, they may not even possess a uniform error probability (UEP) property, so in principle, code design requires an exhaustive search over all error events. Also, in general, CGD of each pair of branches requires calculation of distances between all codeword pairs. In this section, we simplify the code design process by exploiting certain symmetries in our codes.

The key result of this section shows that a large number of calculations can be bypassed, because despite the lack of UEP, many of the distances remain symmetric.

Theorem 1: For $M$-ary (M)PSK modulations, the distances between two converging trellis paths are invariant to the converging state, i.e., $\mathrm{D}\left(\mathcal{T}_{m, 0}, \mathcal{T}_{n, 0}\right)=\mathrm{D}\left(\mathcal{T}_{m, i}, \mathcal{T}_{n, i}\right) \forall m, n, \forall i$. Furthermore, this distance can be calculated by considering only one reference codeword, that is, for any $\mathbf{X} \in \mathcal{T}_{m, 0}$

$$
\mathrm{D}\left(\mathcal{T}_{m, 0}, \mathcal{T}_{n, 0}\right)=\mathrm{D}\left(\mathbf{X}, \mathcal{T}_{n, 0}\right) .
$$

To prove this result, we need the following lemma, which shows that OSTBC codewords (for MPSK) can be mapped to one another by simple pre- and postmultiplication by diagonal matrices.

Lemma 2: Assuming a constant-modulus (MPSK) modulation, for any two OSTBC codewords $\mathbf{X}_{1}, \mathbf{X}_{2} \in \mathcal{T}$ there exist unitary matrices $\mathbf{V}$ and $\mathbf{W}$ such that $\mathbf{X}_{2}=\mathbf{V} \mathbf{X}_{1} \mathbf{W}$. The transform matrices obviously depend on the codewords.

Proof: First consider $L_{t}=2$, where

$$
\mathbf{X}_{1}=\left(\begin{array}{cc}
s_{0} & s_{1} \\
-s_{1}^{*} & s_{0}^{*}
\end{array}\right)
$$

where $s_{0}$ and $s_{1}$ are MPSK symbols. Take any other codeword $\mathbf{X}_{j} \in \mathcal{T}$ with two symbols $s_{0}^{\prime}=s_{0} e^{j \theta}$ and $s_{1}^{\prime}=s_{1} e^{j \phi}$ where $\theta$ and $\phi$ are multiples of $2 \pi / M$. Then

$$
\mathbf{X}_{2}=\left(\begin{array}{cc}
e^{j(\theta+\phi) / 2} & 0 \\
0 & e^{-j(\theta+\phi) / 2}
\end{array}\right) \mathbf{X}_{1}\left(\begin{array}{cc}
e^{j(\theta-\phi) / 2} & 0 \\
0 & e^{-j(\theta-\phi) / 2}
\end{array}\right)
$$

For general $L_{t}$, each entry of the STBC codeword is either a modulation symbol or its conjugate, thus the mapping between two OSTBC codewords consists of elementwise phase change on the codeword matrix. Elementwise multiplication of a matrix can be accomplished via multiplying rows and columns of the matrix by scalars. This, in turn, is accomplished by left-multiplication by a diagonal matrix (multiplies rows by diagonal elements) and right-multiplication by another diagonal matrix (multiplies columns by diagonal elements). Thus the mapping of one OSTBC codeword to another is always possible by leftand right-multiplication by diagonal matrices.

\section{Proof: (Theorem 1)}

$$
\begin{aligned}
\mathrm{D}\left(\mathcal{T}_{m, 0}, \mathcal{T}_{n, 0}\right) & =\mathrm{D}\left(\mathbf{V}_{i} \mathcal{T}_{m, 0} \mathbf{W}_{i}, \mathbf{V}_{i} \mathcal{T}_{n, 0} \mathbf{W}_{i}\right) \\
& =\mathrm{D}\left(\mathbf{V}_{i} \mathcal{T}_{m, 0} \mathbf{W}_{i}, \mathbf{V}_{i} \mathcal{T}_{m, 0} \mathbf{U}_{n} \mathbf{W}_{i}\right) \\
& =\mathrm{D}\left(\mathbf{V}_{i} \mathcal{T}_{m, 0} \mathbf{W}_{i}, \mathbf{V}_{i} \mathcal{T}_{m, 0} \mathbf{W}_{i} \mathbf{U}_{n}\right) \\
& =\mathrm{D}\left(\mathcal{T}_{m, i}, \mathcal{T}_{n, i}\right)
\end{aligned}
$$

where $\mathbf{V}_{i}$ and $\mathbf{W}_{i}$ are codeword transform matrices in the sense of Lemma 2. Equation (3) holds because unitary transforms are distance-preserving, and (4) holds due to commutativity of diagonal matrices $\mathbf{U}_{n}$ and $\mathbf{W}_{i}$. To get the second part of the result, we can write

$$
\mathrm{D}\left(\mathcal{T}_{m, 0}, \mathcal{T}_{n, 0}\right)=\min _{\mathbf{X}_{i} \in \mathcal{T}_{m, 0}} \mathrm{D}\left(\mathbf{X}_{i}, \mathcal{T}_{n, 0}\right)
$$

However, $\mathrm{D}\left(\mathbf{X}_{i}, \mathcal{T}_{n, 0}\right)$ is the same for all $\mathbf{X}_{i} \in \mathcal{T}_{m, 0}$ because

$$
\begin{aligned}
\mathrm{D}\left(\mathbf{X}_{1}, \mathcal{T}_{n, 0}\right) & =\mathrm{D}\left(\mathbf{X}_{1}, \mathcal{T}_{m, 0} \mathbf{U}_{n}\right) \\
& =\min _{i=0, \ldots, M-1} \mathrm{D}\left(\mathbf{X}_{1}, \mathbf{V}_{i} \mathbf{X}_{1} \mathbf{W}_{i} \mathbf{U}_{n}\right) \\
& =\min _{i=0, \ldots, M-1} \mathrm{D}\left(\mathbf{V}_{j} \mathbf{X}_{1} \mathbf{W}_{j}, \mathbf{V}_{j} \mathbf{V}_{i} \mathbf{X}_{1} \mathbf{W}_{i} \mathbf{U}_{n} \mathbf{W}_{j}\right) \\
& =\min _{i=0, \ldots, M-1} \mathrm{D}\left(\mathbf{X}_{j}, \mathbf{V}_{i} \mathbf{X}_{j} \mathbf{W}_{i} \mathbf{U}_{n}\right) \\
& =\mathrm{D}\left(\mathbf{X}_{j}, \mathcal{T}_{m, 0} \mathbf{U}_{n}\right) \\
& =\mathrm{D}\left(\mathbf{X}_{j}, \mathcal{T}_{n, 0}\right)
\end{aligned}
$$

where in (7), we have used the property that if $\mathbf{Y}=\mathbf{V X W}$ for some $\mathbf{X}, \mathbf{Y} \in \mathcal{T}_{m, 0}$, then $\mathbf{V Z W} \in \mathcal{T}_{m, 0}$ for all $\mathbf{Z} \in \mathcal{T}_{m, 0}$.

The above results allow easier CGD calculations. Consider a section of a trellis with length two in Fig. 1(b), and consider events $\mathcal{E}_{i}$ that start at State 0 , go to State $i$, and terminate on State 0 , i.e., $\mathcal{E}_{i}=\mathcal{T}_{0, i} \times \mathcal{T}_{i, 0}$. There may be multiple such events, because there may be parallel paths. Likewise, define $\mathcal{E}_{j}=\mathcal{T}_{0, j} \times \mathcal{T}_{j, 0}$. The distance between $\mathcal{E}_{i}$ and $\mathcal{E}_{j}$ is defined as

$$
\mathrm{D}\left(\mathcal{E}_{i}, \mathcal{E}_{j}\right)=\min _{(\mathbf{X}, \tilde{\mathbf{X}}) \in \mathcal{E}_{i},(\mathbf{Y}, \tilde{\mathbf{Y}}) \in \mathcal{E}_{j}} \operatorname{det}(\mathbf{A}(\mathbf{X}, \mathbf{Y})+\mathbf{A}(\tilde{\mathbf{X}}, \tilde{\mathbf{Y}})) .
$$

Knowing that for positive semidefinite matrices $\operatorname{det}\left(\mathbf{A}_{1}+\right.$ $\left.\mathbf{A}_{2}\right) \leq \operatorname{det}\left(\mathbf{A}_{1}\right)+\operatorname{det}\left(\mathbf{A}_{2}\right)$, we can bound the distance

$$
\begin{aligned}
\mathrm{D}\left(\mathcal{E}_{i}, \mathcal{E}_{j}\right) \leq & \min _{(\mathbf{X}, \tilde{\mathbf{X}}) \in \mathcal{E}_{i},(\mathbf{Y}, \tilde{\mathbf{Y}}) \in \mathcal{E}_{j}} \operatorname{det}(\mathbf{A}(\mathbf{X}, \mathbf{Y}))+\operatorname{det}(\mathbf{A}(\tilde{\mathbf{X}}, \tilde{\mathbf{Y}})) \\
= & \min _{(\mathbf{Y}, \tilde{\mathbf{Y}}) \in \mathcal{E}_{j}} \operatorname{det}\left(\mathbf{A}\left(\mathbf{X}_{0}, \mathbf{Y}\right)\right) \\
& +\min _{(\mathbf{Y}, \tilde{\mathbf{Y}}) \in \mathcal{E}_{j}} \operatorname{det}\left(\mathbf{A}\left(\tilde{\mathbf{X}}_{0}, \tilde{\mathbf{Y}}\right)\right)
\end{aligned}
$$

where $\left(\mathbf{X}_{0}, \tilde{\mathbf{X}}_{0}\right)$ is an arbitrary codeword in $\mathcal{E}_{i}$. The simplification is achieved by invoking Theorem 1: the distance of two sets is identical to the distance of one set to an arbitrary codeword of the other. Finally, we identify the dominant error event by finding the minimum of $\mathrm{D}\left(\mathcal{E}_{i}, \mathcal{E}_{j}\right)$ over all pairs $\left(\mathcal{E}_{i}, \mathcal{E}_{j}\right)$. 
This result is easily extended to partially connected trellises where dominant error events can have length greater than two. In that case, the CGD is bounded by

$$
\operatorname{det}\left(\sum_{i=1}^{k} \mathbf{A}_{i}\right) \leq \sum_{i=1}^{k} \operatorname{det}\left(\mathbf{A}_{i}\right) .
$$

In this case, we bound the CGD by the distances of the diverging and converging paths $\operatorname{det}\left(\mathbf{A}_{1}\right)$ and $\operatorname{det}\left(\mathbf{A}_{k}\right)$, respectively, because the contribution of the interior trellis sections to the CGD is generally unclear, a phenomenon familiar from TCM design [3]. Therefore, only the contribution of the beginning and end trellis sections are used in the cost function, leading to a result similar to (8).

The developments in this section were geared towards generality and insights, thus only bounds were obtained. However, for the special case of the fully connected trellis with length-two error event, it is possible to obtain a precise calculation, which is given in the Appendix.

\section{Code Design ExAMPLes}

We now proceed with specific code examples, simulations, and comparisons with codes in the literature. In our simulations, a frame consists of 130 transmissions, and the number of receive antennas is one. We have extensively used the design tools that we developed in Section II to reduce the search space. While in the literature, the search over the space of codes is based on CGD criterion, we choose a partial union-bound criterion which takes into account the multiplicities and provides better codes. In the trellises demonstrating our design examples (Fig. 1(c), (d), and (e)), we follow the signaling notation of [1].

Fig. 1(c) shows a 4-state fully connected trellis designed for BPSK modulation, full-rate $1 \mathrm{~b} / \mathrm{s} / \mathrm{Hz}$, and $L_{t}=2$. We designed the code to maximize the minimum CGD of events with length two, the minimum length error event. The transforms (rotations) for states 1,2 , and 3 are $\mathbf{U}_{1}=\operatorname{diag}(-j, j), \mathbf{U}_{2}=\operatorname{diag}(j,-1)$, and $\mathbf{U}_{3}=\operatorname{diag}(-1,-j)$. Fig. 2 shows the frame-error probability versus SNR for our 2-state Fig. 1(a) and 4-state code Fig. 1(c), both labeled as new, in slow fading and compare them with the 2-state and 4-state codes given in [1] (labeled as JS). Our 2-state code outperforms JS by about $1 \mathrm{~dB}$ and performs the same as the JS 4-state code.

Fig. 3 compares our proposed 4-state code and the JS 4-state code in slow and fast fading. Since the new 4-state code of Fig. 1(c) does not have parallel branches, it enjoys higher time diversity, and thus outperforms JS significantly in fast fading.

Fig. 1(d) shows the 4-state trellis designed for quaternary (Q)PSK, full-rate $2 \mathrm{~b} / \mathrm{s} / \mathrm{Hz}$, using two transmit antennas. The structure of our 4-state trellis is the same as the trellis in [1] and [2] (JS, SF). The only difference is in the rotation U. Our rotation is $\mathbf{U}=\operatorname{diag}\left(e^{j 3 \pi / 4}, e^{-j 3 \pi / 4}\right)$, but the rotation in [1] and [2] is $\mathbf{U}=\operatorname{diag}\left(e^{j \pi}, 1\right)$. A gain of $0.4 \mathrm{~dB}$ over the $\mathrm{JS}, \mathrm{SF}$ code is achieved.

Our 8-state QPSK trellis is shown in Fig. 1(e), whose performance is slightly better than the 16-state code given in [2]. The transformation matrices for our 8-state trellis is as follows: $\mathbf{U}_{i}=\operatorname{diag}\left(e^{j \theta_{i} \pi}, e^{j \phi_{i} \pi}\right)$, where the pairs of $\left(\theta_{i}, \phi_{i}\right)$ for the

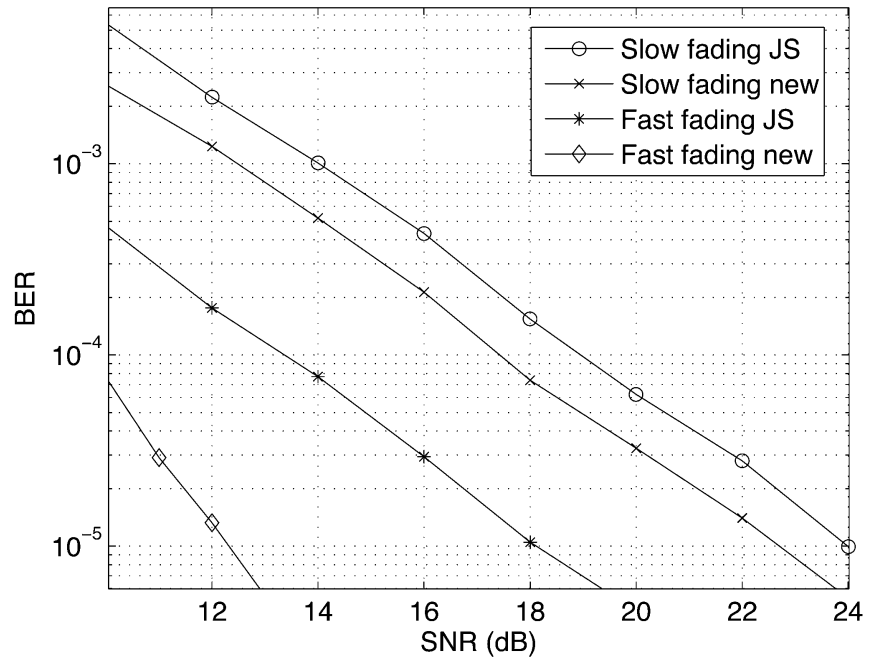

Fig. 3. $L_{t}=2$, 4-state BPSK codes in slow and fast fading.

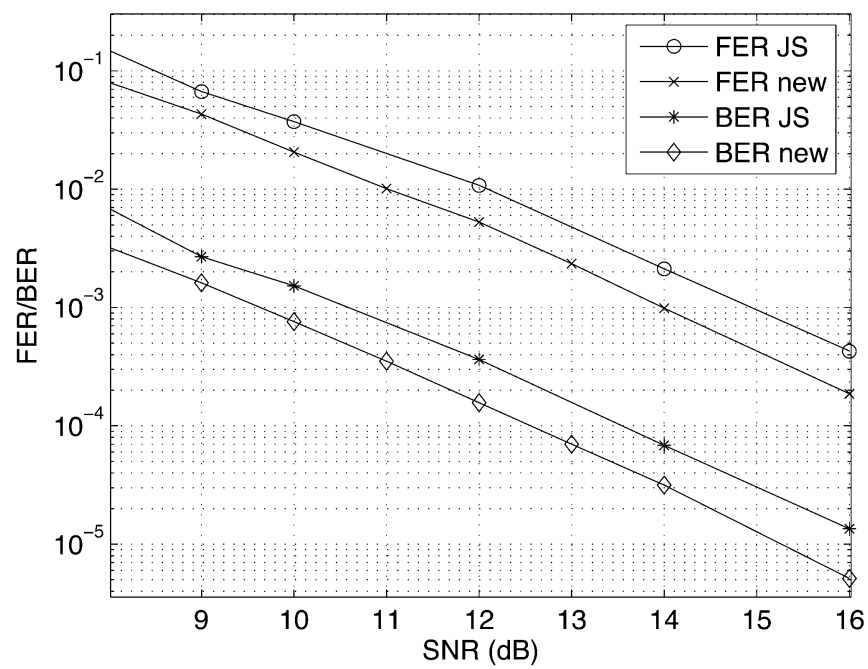

Fig. 4. $L_{t}=4$, two-state BPSK code in slow fading.

states $i=1, \ldots, 7$ are, respectively, $(7 / 4,5 / 4),(3 / 2,3 / 2),(5 / 4$, $3 / 4),(5 / 4,1 / 4),(1,3 / 2),(3 / 4,7 / 4),(1 / 2,1)$.

Now consider four transmit antennas. Fig. 4 shows the the frame-error rate and bit-error rate for a simple 2-state trellis code with four transmit antennas in slow fading. For this code, $\mathbf{U}=$ $\operatorname{diag}\left(e^{j 3 \pi / 2}, e^{j \pi / 2}, e^{j 3 \pi / 2}, e^{j \pi / 2}\right)$. This code gives about $1 \mathrm{~dB}$ gain over the code given in [1], which also outperforms the code in [4] which uses a quasi-orthogonal code.

The gains for QPSK and higher order constellations are somewhat smaller. In Fig. 5, we show 4-state codes for two transmit antennas and QPSK constellations. The structure of our 4-state trellis is the same as the trellis in [1] and [2] (JS, SF). The only difference is in the rotation $\mathbf{U}$.

As a final note, we mention that the decoding complexity is essentially unaffected by rotations $\mathbf{U}$, because a coherent receiver can merge the diagonal matrix $\mathbf{U}$ into the channel matrix. Specifically, the received signal is $\mathbf{R}=\sqrt{\rho} \mathbf{X U H}+\mathbf{N}$. The effective channel gain $\tilde{\mathbf{H}}=\mathbf{U H}$ has the same statistics as $\mathbf{H}$, since $\mathbf{U}$ is unitary. From the receiver point of view, $\tilde{\mathbf{H}}$ is the 


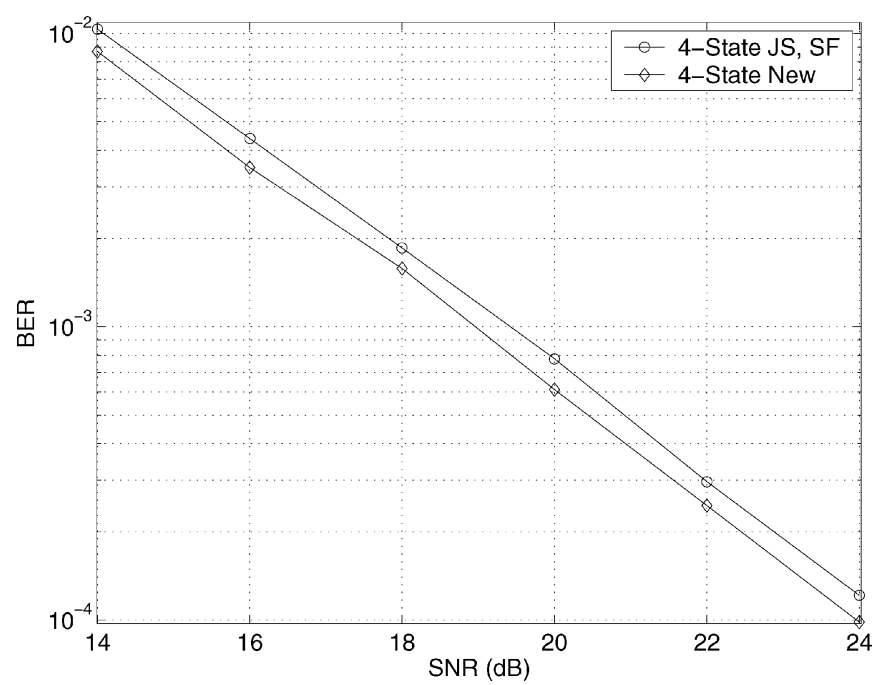

Fig. 5. $L_{t}=2$,4-state QPSK code in slow fading.

effective channel gain matrix. The complexity consists of recalculation of $\tilde{\mathbf{H}}$ whenever CSI is updated.

\section{CONCLUSION}

We propose a generalization of the codes known as superorthogonal codes or, alternatively, STC-TCM codes. Our generalization extends the number of allowable rotations, yielding more powerful codes. We present several properties of codewords and set partitions so that the design process can be simplified. Simulations demonstrate the performance of our generalized codes.

\section{APPENDIX}

Consider a section of a trellis with length two in Fig. 1(b), and denote the set of events starting at state $k$, ending at state $p$, and passing through state $i$ with $\mathcal{E}_{k, i, p}$, i.e., $\mathcal{E}_{k, i, p}=\mathcal{T}_{k, i} \times \mathcal{T}_{i, p}$.
Likewise, define $\mathcal{E}_{k, j, p}=\mathcal{T}_{k, j} \times \mathcal{T}_{j, p}$. The distance between $\mathcal{E}_{k, i, p}$ and $\mathcal{E}_{k, j, p}$ is defined as

$$
\mathrm{D}\left(\mathcal{E}_{k, i, p}, \mathcal{E}_{k, j, p}\right)=\min \operatorname{det}(\mathbf{A}(\mathbf{X}, \mathbf{Y})+\mathbf{A}(\tilde{\mathbf{X}}, \tilde{\mathbf{Y}}))
$$

where the minimum is taken over

$$
\mathbf{X} \in \mathcal{T}_{k, i}, \tilde{\mathbf{X}} \in \mathcal{T}_{i, p}, \mathbf{Y} \in \mathcal{T}_{k, j}, \tilde{\mathbf{Y}} \in \mathcal{T}_{j, p} .
$$

To simplify the expression (thus saving computation), we note that

$$
\begin{aligned}
\mathbf{A}(\mathbf{X}, \mathbf{Y}) & =(\mathbf{X}-\mathbf{Y})(\mathbf{X}-\mathbf{Y})^{H} \\
& =(\overline{\mathbf{X}}-\overline{\mathbf{Y}}) \mathbf{U}_{k} \mathbf{U}_{k}^{H}(\overline{\mathbf{X}}-\overline{\mathbf{Y}})^{H} \\
& =\mathbf{A}(\overline{\mathbf{X}}, \overline{\mathbf{Y}})
\end{aligned}
$$

where $\overline{\mathbf{X}} \in \mathcal{T}_{0, i}$ and $\overline{\mathbf{Y}} \in \mathcal{T}_{0, j}$, which means the starting state $k$ can be set to a fixed state, e.g., state 0 , without loss of generality, i.e., $\mathrm{D}\left(\mathcal{E}_{k, i, p}, \mathcal{E}_{k, j, p}\right)=\mathrm{D}\left(\mathcal{E}_{0, i, p}, \mathcal{E}_{0, j, p}\right)$. Since the set partitioning for $\mathcal{T}_{0}$ is carried out before finding the rotations, this term is calculated only once. Therefore, in the calculation of (10), only the second term involves the rotation matrices $\mathbf{U}_{i}$ and $\mathbf{U}_{j}$.

The dominant error event is the minimum of $\mathrm{D}\left(\mathcal{E}_{0, i, p}, \mathcal{E}_{0, j, p}\right)$ over all pairs $\left(\mathcal{E}_{0, i, p}, \mathcal{E}_{0, j, p}\right)$. In the design process, a search is conducted to find rotations that maximize the minimum distance obtained above.

\section{REFERENCES}

[1] H. Jafarkhani and N. Seshadri, "Super-orthogonal space-time trellis codes," IEEE Trans. Inf. Theory, vol. 49, no. 4, pp. 937-950, Apr. 2003.

[2] S. Siwamogsatham and M. P. Fitz, "Improved high-rate space-time codes from expanded STB-MTCM construction," IEEE Trans. Inf. Theory, vol. 51, no. 12, pp. 4173-4183, Dec. 2005.

[3] G. Ungerböck, "Trellis-coded modulation with redundant signal sets: Part I and II," IEEE Commun. Mag., vol. 25, no. 2, pp. 5-21, Feb. 1987.

[4] H. Jafarkhani and N. Hassanpour, "Super-quasi-orthogonal space-time trellis codes for four transmit antennas," IEEE Trans. Wireless Commun., vol. 4, no. 1, pp. 215-227, Jan. 2005. 